\title{
Multi-Fresnel lenses pumping approach for improving high-power Nd:YAG solar laser beam quality
}

\author{
Dawei Liang* and Joana Almeida \\ CEFITEC, Departamento de Física, FCT, Universidade Nova de Lisboa, 2829-516 Campus de Caparica, Portugal \\ ${ }^{*}$ Corresponding author: dl @fct.unl.pt
}

Received 9 April 2013; revised 3 June 2013; accepted 12 June 2013; posted 14 June 2013 (Doc. ID 188584); published 0 MONTH 0000

\begin{abstract}
To significantly improve the present-day high-power solar laser beam quality, a three-stage multiFresnel lenses approach is proposed for side-pumping either a Nd:YAG single-crystal or a core-doped $\mathrm{Sm}^{3+} \mathrm{Nd}$ :YAG ceramic rod. Optimum pumping and laser beam parameters are found through ZEMAX and LASCAD numerical analysis. The proposed scheme offers a uniform absorption profile along the rod. $167 \mathrm{~W}$ laser power can be achieved, corresponding to $29.3 \mathrm{~W} / \mathrm{m}^{2}$ collection efficiency. High brightness figure of merit of $8.34 \mathrm{~W}$ is expected for the core-doped rod within a convex-concave resonator, which is 1300 times higher than that of the most-recent high-power solar laser. () 2013 Optical Society of America

OCIS codes: (140.0140) Lasers and laser optics; (140.3580) Lasers, solid-state; (140.3410) Laser resonators; (140.3530) Lasers, neodymium; (350.6050) Solar energy.

http://dx.doi.org/10.1364/AO.99.099999
\end{abstract}

\section{Introduction}

Directly converting broadband solar radiation into coherent and narrowband laser radiation has gained an ever-increasing importance in recent years [1]. Compared to electrically powered lasers, a solar laser is much simpler and more reliable due to the complete elimination of the electrical power generation and conditioning equipments. Solar pumping of solid-state lasers saves two energy conversion steps, and thus is inherently more efficient. This technology has a large potential for many applications, e.g., high-temperature materials processing, free space laser communications, space to Earth power transmission, and so on.

The first solar-pumped solid-state laser was reported by Young in 1966 [2]. Since then, researchers have been improving constantly the solar laser performance [ $\underline{3}-\underline{11}]$. The progress with Fresnel

$1559-128 \mathrm{X} / 13 / 160001-01 \$ 15.00 / 0$

(C) 2013 Optical Society of America lenses and chromium co-doped Cr:Nd:YAG ceramic laser medium has revealed a promising future for the renewable magnesium-hydrogen cycle $[\underline{7}, \underline{8}]$. Despite the interests in $\mathrm{Cr}: \mathrm{Nd}: \mathrm{YAG}$ ceramic medium $[8,12]$, the most widely used Nd:YAG laser material has been demonstrated as an excellent material under solar pumping because of its superior characteristic on thermal conductivity, high quantum efficiency, and mechanical strength compared to other host materials [2-6, $9-11]$. Significant solar laser collection efficiencies have been achieved with different $\mathrm{Nd}$ :YAG single-crystal rods. $19.3 \mathrm{~W} / \mathrm{m}^{2}$ collection efficiency has been reported by us in 2011 [9] by utilizing an economical Fresnel lens and a small diameter Nd:YAG single-crystal rod. The most recent Nd: YAG solar-pumped laser with a liquid light-guide lens has produced record-high collection efficiency of $30.0 \mathrm{~W} / \mathrm{m}^{2}$, in 2012 [11]. Despite the strong desire to achieve high collection efficiency, much more attention should also be paid to the solar laser beam quality in order to attain tight focusing, which is an essential issue for most laser applications. 
Although the most efficient laser systems have end-pumping approaches $[\underline{8}, \underline{9}, \underline{11}]$, side-pumping is an effective configuration for power scaling as it gives uniform absorption along the rod axis and spreads the absorbed power along the laser medium, reducing hence the associated thermal loading problems. Besides, the free access to both rod ends allows the optimization of more laser resonator parameters, capable of improving largely laser beam quality. The solar laser beam brightness of a side-pumping configuration can hence be much higher than that of end-pumping configuration. Brightness is one of the most important parameters of a laser beam. It is given by the laser power divided by the product of the beam spot area and its solid angle divergence. This product is proportional to the square of beam quality factor $M^{2}$. Brightness figure of merit B is thus defined [6] as the ratio between laser power and the product of $M_{x}^{2}$ and $M_{y}^{2}$. Record-high brightness figure of merit of $0.29 \mathrm{~W}$ has been registered in 2011 by side-pumping a $4 \mathrm{~mm}$ diameter $30 \mathrm{~mm}$ length $\mathrm{Nd}$ : YAG single-crystal rod through a light guide/modified 2D-CPC cavity [10]. Despite the successful production of a $120 \mathrm{~W}$ high-power Nd:YAG solar laser beam with end-pumping configuration, very large $M_{x}^{2}=M_{y}^{2}=137$ beam quality factors have also been reported [11], resulting in the brightness figure of merit of only $0.0064 \mathrm{~W}$.

To largely improve the laser beam quality of highpower solar-pumped solid-state lasers, an alternative solar-pumping approach composed of multiple Fresnel lenses is put forward in this paper for side-pumping either the Nd:YAG single-crystal rod or the core-doped ceramic $\mathrm{Sm}^{3+} \mathrm{Nd}$ :YAG rod. Compared to our previous pumping scheme for solarpumped disk laser [13], high efficiency solar energy collection and concentration can be achieved through the spatial combination of three stages, each composed of 32 pairs of narrow plane mirrors and Fresnel lenses, which are symmetrically aligned around the Nd:YAG laser rod. The concentrated solar radiation from the Fresnel lenses are finally compressed along the Nd:YAG rod through either a cylindrical or a spherical fused silica lens. Optimum optical pumping conditions are found through ZEMAX software. Nonsequential ray-tracing is performed to analyze both the absorption efficiencies and the absorbed pump flux distributions within the Nd:YAG single-crystal rods of different dimensions. Optimized solar laser beam parameters are found through LASCAD numerical analysis. A $9 \mathrm{~mm}$ diameter, $30 \mathrm{~mm}$ length $\mathrm{Nd}$ :YAG single-crystal rod, pumped through the cylindrical lens, presents the most uniform absorbed pump flux distribution along the rod axis. High multimode solar laser power of $146 \mathrm{~W}$ is expected in this case. This value can also be further improved by pumping shorther laser rods. Besides the analysis of traditional concave-concave resonators, the $M^{2}$ factors and the brightness figure of merit are also numerically analyzed for the convexconcave resonators. Optical resonators employing one convex and one concave mirror increase the volume of fundamental mode, suppressing higher order resonator modes, and beam quality improves $[6,14,15]$. Also, low sensitivity to perturbations has been obtained in comparison to other resonator types [14]. By pumping the $9 \mathrm{~mm}$ diameter, $30 \mathrm{~mm}$ length $\overline{\mathrm{Nd}}$ :YAG single-crystal rod within the convexconcave resonator, $4.0 \mathrm{~W}$ brightness figure of merit is expected. This value is 6.8 times higher than that achieved with the concave-concave resonator for the same rod. The solar laser performances of core-doped ceramic Nd:YAG rods with $\mathrm{Sm}^{3+}$-doped YAG cladding are finally studied and compared to that of the single-crystal rods within either the concaveconcave or the convex-concave resonators respectively. The solar laser performances can be enhanced by the use of both core-doped and the convex-concave cavity techniques. On the one hand, $29.3 \mathrm{~W} / \mathrm{m}^{2}$ collection efficiency can be achieved with an $8 \mathrm{~mm}$ diameter, $20 \mathrm{~mm}$ length core-doped Nd:YAG ceramic rod. On the other hand, $8.34 \mathrm{~W}$ brightness figure of merit is attainable with an $8 \mathrm{~mm}$ diameter, $30 \mathrm{~mm}$ length Nd:YAG core-doped rod, which is more than 1300 times that of the most recent $120 \mathrm{~W}$ high-power solar-pumped laser [11].

(a)

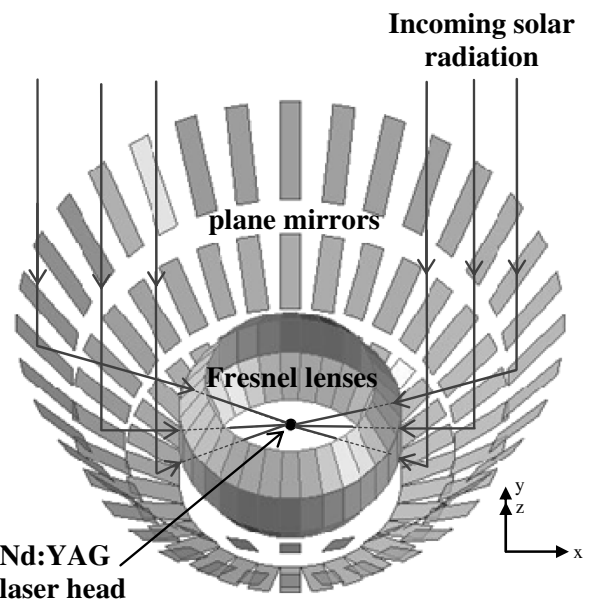

(b)

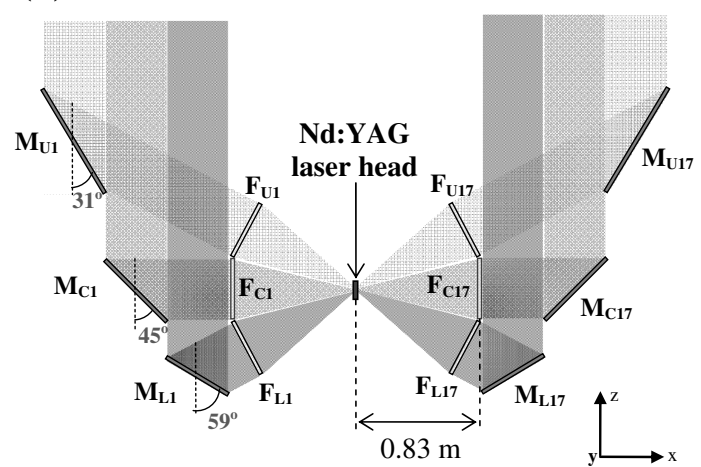

Fig. 1. (a) Multiplane mirrors: Fresnel lenses solar pumping approach for Nd:YAG solar laser. (b) Cross-sectional side-view of the solar light collection and concentration system. 


\section{Multi-Fresnel Lenses Pumping Approach for} Emitting High-Quality Nd:YAG Solar Laser Beam

The proposed solar laser pumping approach in Fig. 1 is formed by three stages of solar energy collection and concentration units, each composed of 32 pairs of narrow plane mirrors and Fresnel lenses for side-pumping the Nd:YAG laser rod located in the central focal zone. The Fresnel lenses are evenly distributed along the $0.83 \mathrm{~m}$ radius virtual sphere around the center of a laser head. The formation of each stage has 32-fold symmetry. Each central stage Fresnel lens $\left(F_{C}\right)$ has a narrow rectangular shape with $0.4 \mathrm{~m}$ height and $0.16 \mathrm{~m}$ width, whereas each upper and lower stage Fresnel lens $\left(F_{U}\right.$ and $\left.F_{L}\right)$ presents a trapezoidal shape with $0.4 \mathrm{~m}$ height, $0.125 \mathrm{~m}$ minor base, and $0.160 \mathrm{~m}$ major base.

The narrow Fresnel lenses permit a tight paraxial focusing in $x-y$ plane, while allowing some power spread along the $z$ axis of the laser rod. This leads to both an efficient and a uniform pump light concentration along the laser rod, as compared to that through square Fresnel lenses. The total solar energy collection area of $5.7 \mathrm{~m}^{2}$ is considered for the proposed scheme.

The Fresnel lenses are made of polymethyl methacrylate (PMMA) material, which is transparent at visible and near infrared wavelengths, but absorbs the infrared radiation beyond $2200 \mathrm{~nm}$ and cut undesirable UV solar radiation below $350 \mathrm{~nm}$. An averaged transmission efficiency of $80.8 \%$ is numerically calculated for each Fresnel lens. Experimental transmission efficiency of $80 \%$ confirms the above numerical analysis result. To redirect the incoming solar radiation toward the Fresnel lenses, each plane mirror is mounted behind its corresponding Fresnel lens, along their common optical axis. The central, upper, and lower stages plane mirrors are inclined at $45^{\circ}, 31^{\circ}$, and $59^{\circ}$ angles, respectively, in relation to its $z$ axis, as indicated in Fig. 1(b). Solar tracking can be achieved by mounting the whole system onto a two-axis heliostat that follows the Sun continuously in direct tracking mode. Considering the terrestrial solar insolation of $950 \mathrm{~W} / \mathrm{m}^{2}$, and $95 \%$ reflectivity of each plane mirror, a total amount of $4115 \mathrm{~W}$ solar power can be delivered to the focal zone. The concentrated solar radiation from the Fresnel lenses is then further compressed to the Nd:YAG laser rod through either the cylindrical or the spherical lens, as shown in Figs. 2(a) and 2(b), respectively.

The incoming light from the central stage plane mirrors-Fresnel lenses $\left(M_{C}-F_{C}\right)$ is compressed onto the center of the laser rod, whereas the upper and lower stage plane mirrors-Fresnel lenses $\left(M_{U}-F_{U}\right.$ and $\left.M_{L}-F_{L}\right)$ are responsible for the concentration of pump light onto both the lower and the upper regions of the rod respectively, as illustrated in Fig. 2. This ensures the uniform solar pump power deposition along the laser rod. The heat load can hence be reduced by increasing rod length. The laser rod is directly cooled by water, which ensures the efficient removal of the generated heat. The IR radiation that does not contribute to lasing is firstly attenuated by the Fresnel lenses and then filtered by both the fused silica lens and cooling water. These materials are also useful for partially preventing UV solarization to the laser rod. Fused silica is an ideal optical material for Nd:YAG laser pumping since it is transparent over the Nd:YAG absorption spectrum. It has a high softening point and is resistant to scratching and thermal shock, which also makes it very suitable for high-power solar pumping. A high optical quality fused silica lens (99.999\%), with either cylindrical or spherical shape, can be manufactured by optical machining and polishing [16]. Both end faces of the Nd:YAG rod are $1064 \mathrm{~nm}$ A $\bar{R}$ coated. The laser resonant cavity is formed by both the $1064 \mathrm{~nm}$ HR mirror and the PR output coupler, as shown in Fig. 2.
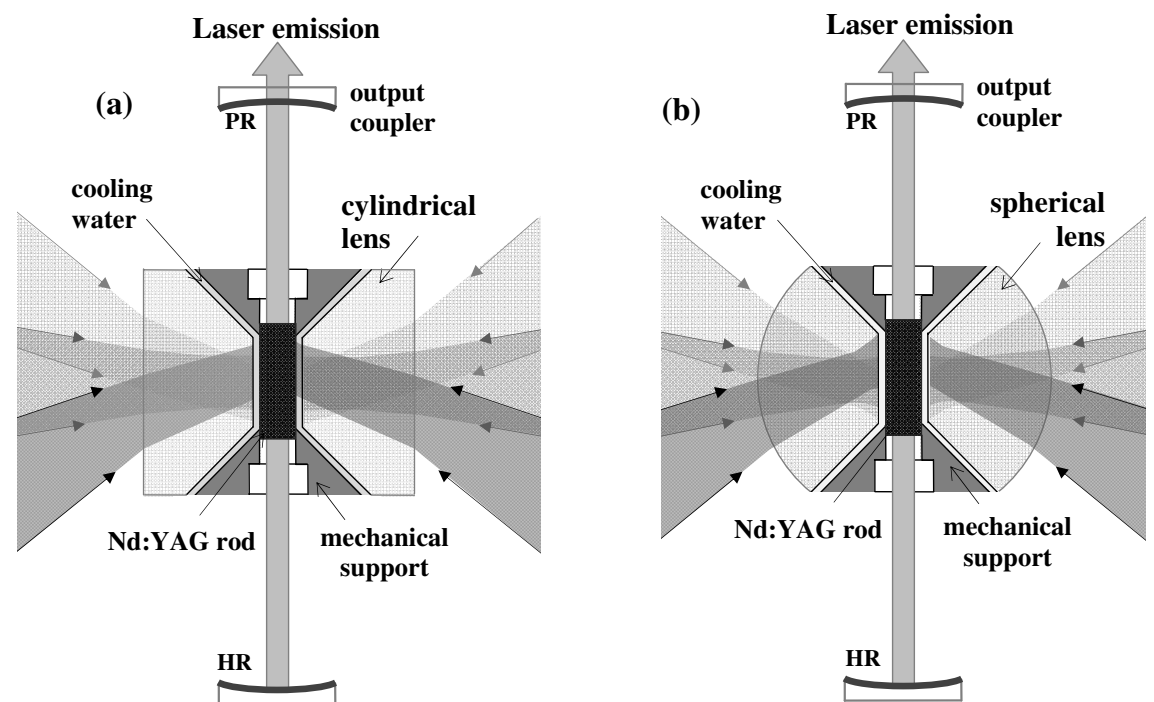

Fig. 2. Pump light concentration onto the Nd:YAG rod through (a) the cylindrical lens and (b) the spherical lens. 


\section{Numerical Analysis of Absorbed Pump Flux} Distributions and Solar Laser Performances with Nd: YAG Single-Crystal Rods

\section{A. Analysis of Both Absorbed Pump Flux Distributions and Absorbed Pump Powers}

All the design parameters of the solar pumping approach are optimized through ZEMAX nonsequential ray-tracing software in order to obtain the most favorable absorbed pump flux distribution within the Nd:YAG rod. The standard solar spectrum for oneand-a-half air mass (AM1.5) [17] is used as the reference data for consulting the spectral irradiance $\left(\mathrm{W} / \mathrm{m}^{2} / \mathrm{nm}\right)$ at each wavelength. The effective pump power of the light source takes into account about $16 \%$ overlap between the absorption spectrum of the Nd:YAG medium and the solar spectrum [18]. The apparent half-angle of $0.27^{\circ}$ subtended by the
Sun [19] is also considered in the analysis. The absorption spectrum of PMMA, fused silica, and water materials are included in ZEMAX numerical data to account for absorption losses.

For 1.0\% Nd:YAG laser medium, 22 absorption peaks are defined in ZEMAX numerical data. All the peak wavelengths and their respective absorption coefficients are added to the glass catalogue for $\mathrm{Nd}$ :YAG material in ZEMAX software. Solar irradiance values for the abovementioned 22 peak absorption wavelengths could be consulted from the standard solar spectra for AM1.5 and saved as source wavelength data. In ray-tracing, the laser rod is divided into a total of 18000 zones. The path length in each zone is found. With this value and the effective absorption coefficient of $1.0 \% \mathrm{Nd}$ :YAG material, the absorbed power within the laser medium can be calculated by summing up the absorbed pump radiation of all zones.

\section{Nd:YAG pumping through the cylindrical lens}
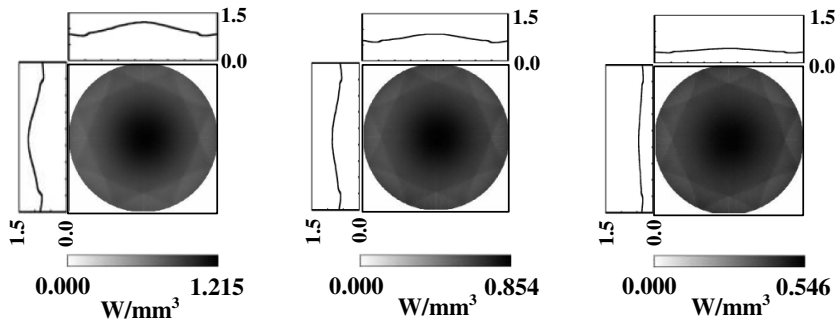

$\mathbf{L}=\mathbf{2 0} \mathbf{~ m m}$

\section{D $_{\text {Nd:YAG }}=9 \mathrm{~mm}$}

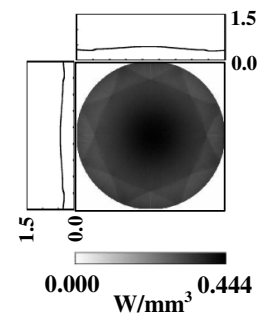

$\mathbf{L}=\mathbf{3 0} \mathbf{~ m m}$

$\mathbf{L}=\mathbf{3 5} \mathbf{~ m m}$

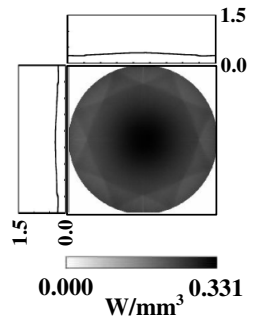

$\mathrm{L}=\mathbf{4 0} \mathrm{mm}$
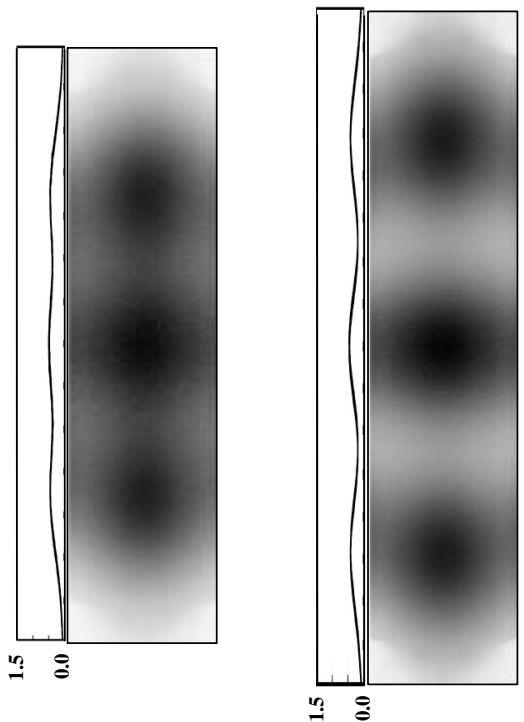

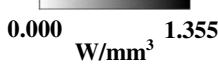

Pabs $=567 \mathrm{~W}$
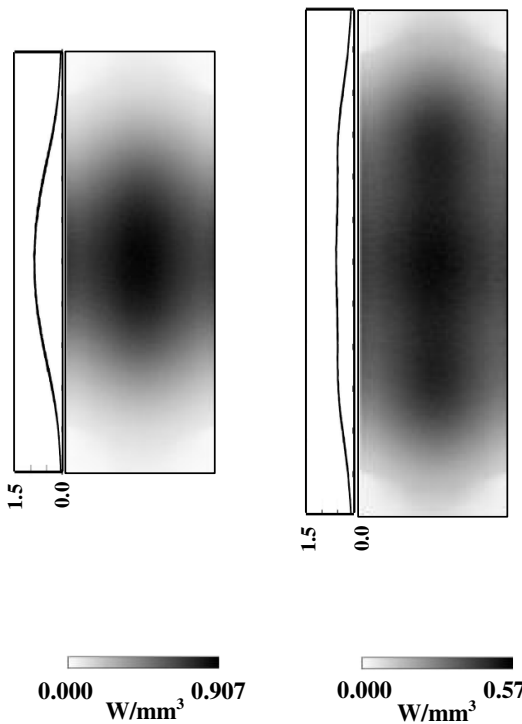

Pabs $=576 \mathrm{~W}$

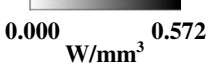

Pabs $=578 \mathrm{~W}$

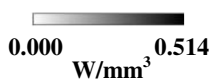

Pabs $=579 \mathrm{~W}$

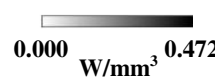

Pabs $=579 \mathrm{~W}$

F3:1 Fig. 3. Absorbed pump flux distributions along both the central and longitudinal cross-sections of the $D_{\mathrm{Nd}: \mathrm{YAG}}=9 \mathrm{~mm}$ single-crystal rods 


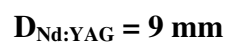

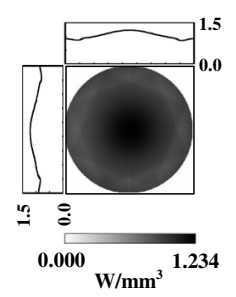

$L=20 \mathrm{~mm}$

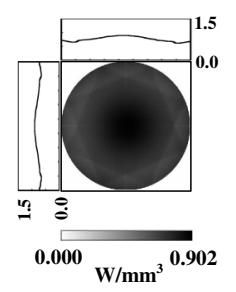

$\mathbf{L}=\mathbf{2 5} \mathrm{mm}$

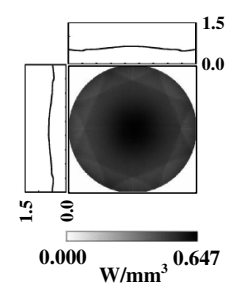

$\mathrm{L}=\mathbf{3 0} \mathrm{mm}$

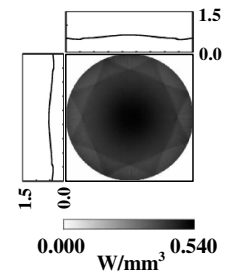

$\mathrm{L}=35 \mathrm{~mm}$

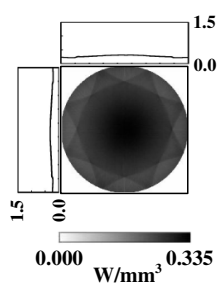

$L=40 \mathrm{~mm}$
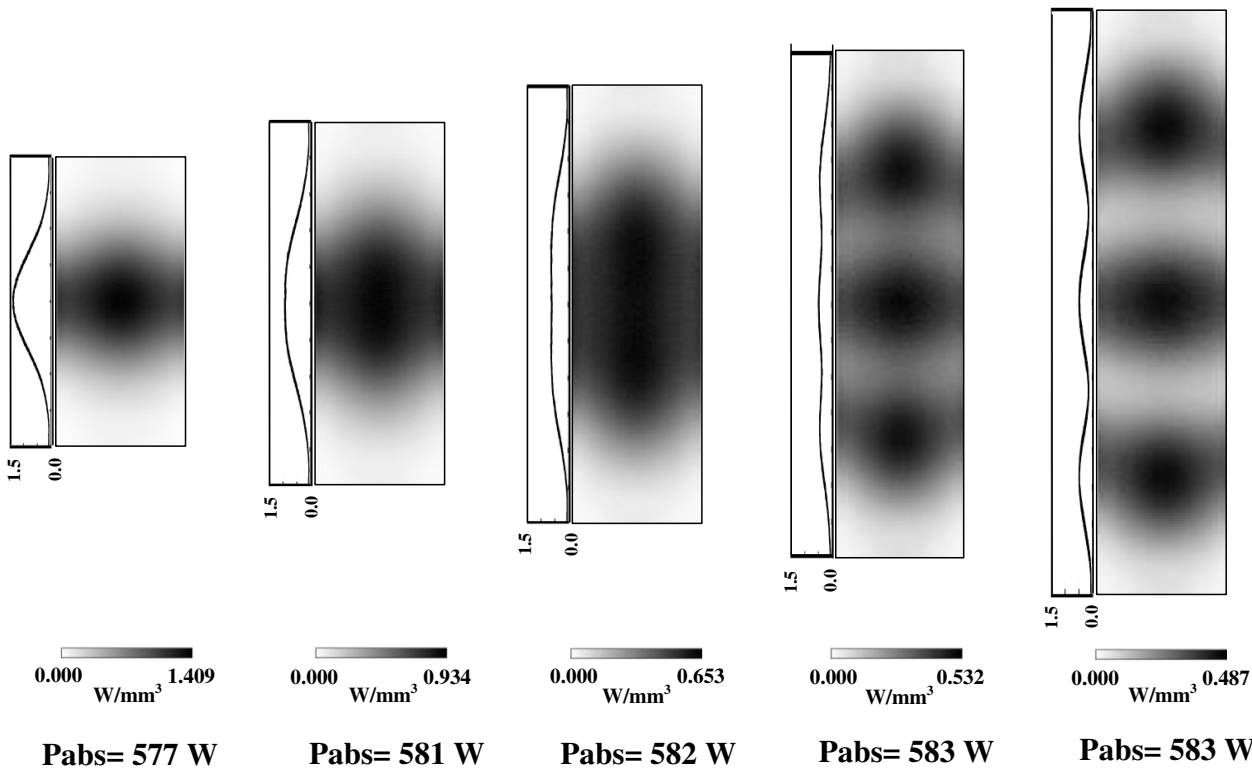

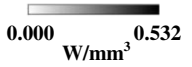

Pabs $=583 \mathrm{~W}$

Pabs $=583 \mathrm{~W}$

F4:1 Fig. 4. Absorbed pump flux distribution along both the central and longitudinal cross-sections of the $D_{\text {Nd:YAG }}=9$ mm single-crystal rods F4:2 with different length, pumped through the spherical lens.

The gray-scale absorbed pump flux distributions within both the central and longitudinal crosssections of the $9 \mathrm{~mm}$ diameter $\left(D_{\mathrm{Nd}: \mathrm{YAG}}\right)$ rods of different length $(L)$, pumped through either the cylindrical or the spherical lens, can now be numerically analyzed, as shown in Figs. $\underline{3}$ and $\underline{4}$, respectively. Black color means near maximum pump absorption for these plots, whereas white means little or no absorption. The absorbed pump power is also given in each case. For each change in both laser rod length and fused silica lens geometry, the mounting positions and angles of the three-stage plane mirrors-Fresnel lenses have to be slightly readapted. Optimized solar pump absorption distribution within the laser rod is hence ensured.

As observed in both Figs. $\underline{3}$ and $\underline{4}$, the $D_{\mathrm{Nd}: \mathrm{YAG}}=$ $9 \mathrm{~mm}, L=30 \mathrm{~mm}$ single-crystal presents the most uniform absorption profile along the rod, mainly through the cylindrical lens, which allows the more widely spread absorbed pump flux. This is very useful for the alleviation of the thermal effects of the active medium. Shorter laser rods present a more centrally peaked absorption profile, resulting in both higher absorbed pump flux and lower absorbed pump power, as compared to that of longer rods. The pumping of Nd:YAG rods longer than $30 \mathrm{~mm}$ leads to the appearance of three small peaks along the crystal. Besides, the absorbed pump power becomes nearly the same for these rods. The adoption of the spherical lens geometry offers more absorbed pump power, specially for the $L=20-25 \mathrm{~mm}$ laser rods, resulting in a slightly higher absorbed pump flux, as compared to that with the cylindrical lens.

\section{B. Analysis of Nd:YAG Single-Crystal Solar Laser Output Powers}

The absorbed pump flux data from the ZEMAX analysis is processed by LASCAD software to study the laser beam parameters of the Nd:YAG singlecrystal rods. In LASCAD analysis the optical resonator is composed of two opposing parallel mirrors at right angles to the axis of the active medium. One end mirror is high reflection coated (HR, 99.98\%). The output coupler is partial reflection coated (PR, variable between $90 \%-99 \%$ in LASCAD analysis). The amount of feedback is determined by the reflectivity of the PR mirrors. Different rod lengths imply different round-trip losses. For example, 
(a)

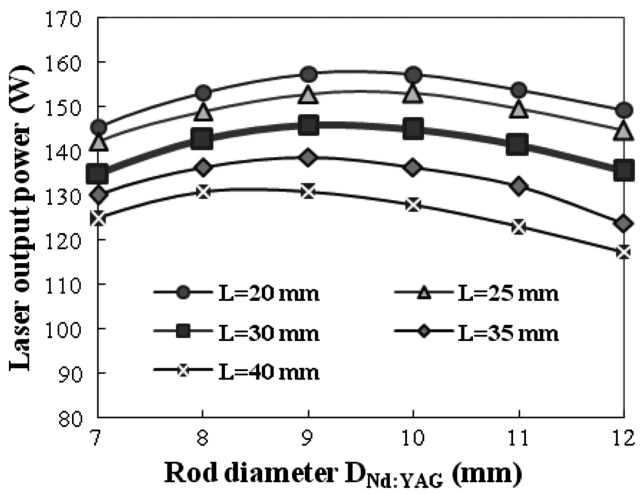

(b) Spherical lens

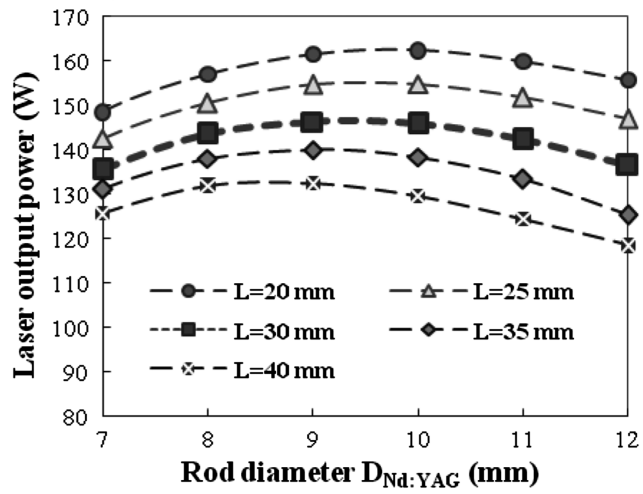

F5:1 Fig. 5. Numerically calculated multimode solar laser power of the Nd:YAG single-crystal rods with different $D_{\text {Nd:YAG }}$ and $L$, pumped F5:2 through (a) the cylindrical lens and (b) the spherical lens.

$3052.4 \%$ round-trip loss is assumed for the $L=30 \mathrm{~mm}$ $306 \mathrm{Nd}: Y A G$ rods in LASCAD analysis. An averaged so307 lar pump wavelength of $660 \mathrm{~nm}$ is also assumed [4]. 308 All the technical parameters and data used 309 in both ZEMAX and LASCAD analysis have 310 already been confirmed by our previous experimen311 312 313 314 315 316

T1:8

T1:9 tal results $[\underline{9}, \underline{10}]$.

Figure 5 gives the comparison between the calculated multimode laser power for different $D_{\mathrm{Nd}: Y A G}$ and $L$ of the single-crystal rods, pumped through either the cylindrical or the spherical lens. To facilitate our comparison with the classical solar laser resonator [6], a symmetric concave-concave resonator with $80 \mathrm{~cm}$ length is adopted in LASCAD software in these cases.

The behavior of the multimode solar laser power as function of both $D_{\mathrm{Nd} \text { :YAG }}$ and $L$ is similar in both cases. It decreases with increased rod length and attain the highest values mostly by the $D_{\mathrm{Nd}: \mathrm{YAG}}=$ 9-10 mm rods, as shown in Fig. 5. The spherical lens geometry offers more laser power, especially for short rods. Maximum multimode laser power of $162.5 \mathrm{~W}$ is numerically achieved by the $D_{\mathrm{Nd} \text { YAG }}=10 \mathrm{~mm}$, $L=20 \mathrm{~mm}$ single-crystal rod, corresponding to $28.5 \mathrm{~W} / \mathrm{m}^{2}$ solar laser collection efficiency. Nevertheless, that value is only slightly more than the maximum laser power of $157.5 \mathrm{~W}$ attained by the $D_{\mathrm{Nd} \text { :YAG }}=9 \mathrm{~mm}, L=20 \mathrm{~mm}$ rod through the cylindrical lens. Besides, for the $L=30-40 \mathrm{~mm}$ rods, the multimode laser power is nearly the same

Table 1. Numerically Calculated Laser Beam Quality Parameters with Nd:YAG Single-Crystal Rod

\begin{tabular}{|c|c|c|}
\hline Resonator Configuration & Concave-Concave & Convex-Concave \\
\hline \multicolumn{3}{|l|}{ Mirror RoC (cm) } \\
\hline Rear & 500 & -50 \\
\hline Output & 500 & 500 \\
\hline Resonator length (cm) & 80 & 80 \\
\hline \multicolumn{3}{|c|}{ Laser beam quality } \\
\hline $\mathrm{M}_{\mathrm{x}}^{2}$ & 15.8 & 6.0 \\
\hline $\mathrm{M}_{\mathrm{v}}^{\mathrm{A}}$ & 15.8 & 6.1 \\
\hline Figure of merit $B(\mathrm{~W})$ & 0.59 & 4.0 \\
\hline
\end{tabular}

for both lens geometries. About $146 \mathrm{~W}$ multimode laser power is achieved by the $D_{\mathrm{Nd}: \mathrm{YAG}}=9 \mathrm{~mm}, L=$ $30 \mathrm{~mm}$ rod pumped through either the cylindrical lens or the spherical lens. Despite the reduction in laser power, the $D_{\mathrm{Nd}: \mathrm{YAG}}=9 \mathrm{~mm}, L=30 \mathrm{~mm}$ rod offers the best compromise between laser power and absorbed pump distribution, as verified in Section 3 .

C. Analysis of Nd:YAG Single-Crystal Solar Laser Beam Quality

Table 1 compares both the $M^{2}$ factors and brightness figure of merit $\mathrm{B}$ numerically achieved by the $D_{\text {Nd:YAG }}=9 \mathrm{~mm}, L=30 \mathrm{~mm} \mathrm{Nd}$ :YAG single-crystal rod pumped through the cylindrical lens, within either the concave-concave or convex-concave resonator.

As shown in Table 1, the concave-concave resonator leads to the largest $M^{2}$ factors and the lowest laser beam brightness. Nevertheless, $0.59 \mathrm{~W}$ brightness figure of merit is numerically achieved, corresponding to $203 \%$ enhancement over the record brightness figure of merit for Nd:YAG solar laser [10]. The solar laser beam quality can be significantly improved by adopting the convex-concave resonator. The $M^{2}$ factors are reduced to $38.6 \%$ of its original value and the brightness figure of merit is therefore 6.8 times higher.

\section{Numerical Analysis of Solar Laser Performances with Core-Doped Nd:YAG Ceramic Rods and its Comparison to that with Nd:YAG Single-Crystal Rods}

Polycrystalline ceramic Nd:YAG laser material can act as host material and offers new possibilities in designing the laser medium with respect to dopant concentration as well as distribution, size, and geometry $[20,21]$. The core-doped $\mathrm{Nd}: \mathrm{YAG}$ ceramic mediums are laser active in the $\mathrm{Nd}^{+3}$-doped core only, and bonded with the same host material either un-doped or doped with a different element that effectively absorbs light at the signal wavelength. This technology, initially developed for diodepumped arrangements, has led to enhanced laser beam brightness compared to conventional singlecrystal rods [22,23]. More recently, core-doped 
ceramic rods have also shown to be promising candidates for solar-pumped solid-state lasers $[13,24]$. Since the rod cross-section is widened by the cladding, wider intensity distributions can be accommodated in the laser active region without truncating its wings. This will lead to a more efficient use of the builtup inversion as the average intensity in the doped part of the laser rod becomes higher. Moreover, the negative impact of diffraction on the laser beam quality should be reduced [22,23].

Among various absorber materials, $\mathrm{Sm}^{3+}$ :YAG is found to be the best candidate to Nd:YAG laser, due to its spectroscopy properties. It has strong absorption at the laser wavelength of $1064 \mathrm{~nm}$ but only little absorption in 500-1000 nm bands [25]. Therefore, the remaining IR radiation can be strongly absorbed, reducing the thermal effects within the Nd:YAG medium. $\mathrm{Sm}^{3+}$ :YAG is also effective in suppressing parasitic oscillations, which limit extraction efficiency and energy scaling of lasers [25-27]. The absorption spectrum of $\mathrm{Sm}^{3+}: \mathrm{YAG}$ is added to the glass catalogue in ZEMAX software.

The influence of the core-doped Nd:YAG rods on the solar laser performance is hence studied for the multi-Fresnel lenses pumping approach. Figure 6 gives the numerically calculated multimode laser powers for the core-doped rods with the same $D_{\text {Nd:YAG }}$ and $L$ of the single-crystal rods, as used in Section 3.

The implementation of core-doped Nd:YAG ceramic rods contributes to the enhancement of the absorbed pump power within the active region of the medium, favoring the production of low-order modes. The maximum multimode laser output power of $167 \mathrm{~W}$ is numerically achieved by the $D_{\mathrm{Nd}: \mathrm{YAG}}=8 \mathrm{~mm}, L=$ $20 \mathrm{~mm}$ core-doped rod. $29.3 \mathrm{~W} / \mathrm{m}^{2}$ collection efficiency is therefore numerically calculated in this case, approaching the record-high collection efficiency for Nd:YAG solar laser [11]. For the $D_{\mathrm{Nd}: \mathrm{YAG}}=8 \mathrm{~mm}$, $L=30 \mathrm{~mm}$ core-doped rods, maximum laser power of $157.8 \mathrm{~W}$ is also achieved, corresponding to $27.7 \mathrm{~W} / \mathrm{m}^{2}$ collection efficiency. An optimized cladding diameter of $17 \mathrm{~mm}$ is found for these cases.

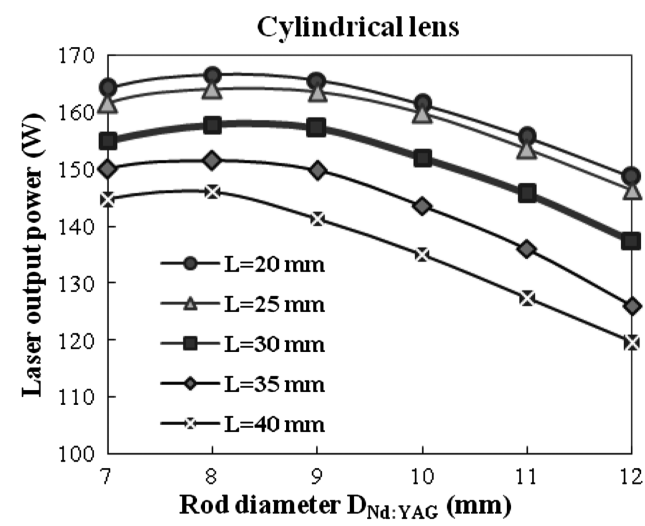

F6:1

$\mathrm{F} 6: 2$

$\mathrm{F} 6: 3$
Fig. 6. Numerically calculated multimode solar laser power of core-doped Nd:YAG ceramic rods with different $D_{\mathrm{Nd} \text { :YAG }}$ and $L$, pumped through the cylindrical lens.

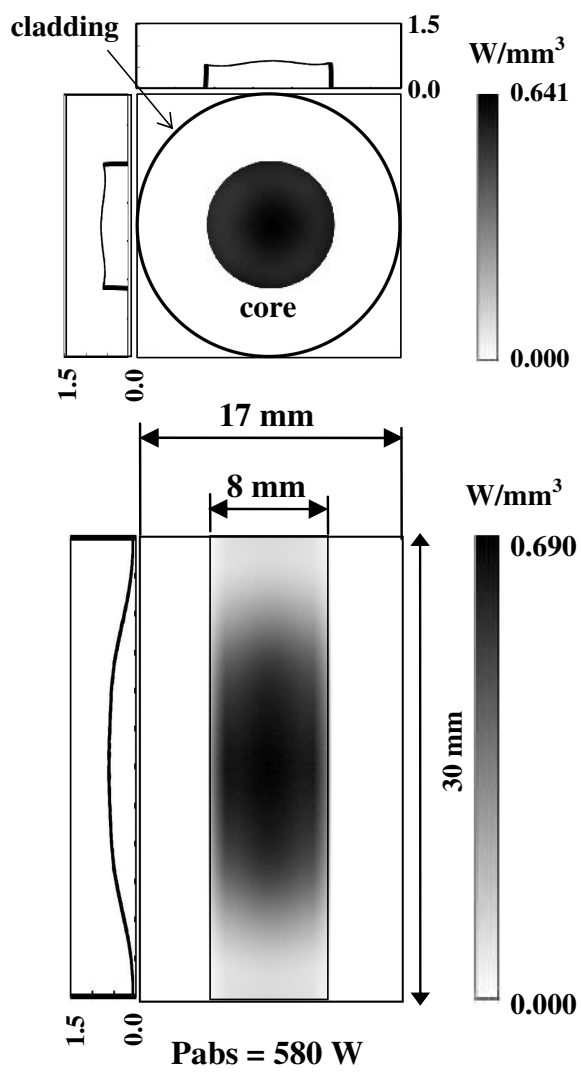

Fig. 7. Absorbed pump flux distribution along both the central and longitudinal cross-sections of the $D_{\mathrm{Nd}: \mathrm{YAG}}=8 \mathrm{~mm}, L=$ $30 \mathrm{~mm}$ core-doped ceramic rod, pumped through the cylindrical lens.

The gray-scale absorbed pump flux distributions within both the central and longitudinal crosssections of the $D_{\mathrm{Nd} \text { Y YAG }}=8 \mathrm{~mm}, L=30 \mathrm{~mm}$ coredoped rod are shown in Fig. 7 . The absorbed pump power is also given.

As observed in Fig. 7, by pumping core-doped $\mathrm{Nd}$ : YAG laser rod through the cylindrical lens, the power deposition is also uniformly spread along the active medium compared to that of the single-crystal rods. Despite the reduced core-diameter of only $8 \mathrm{~mm}$, as compared to the $D_{\mathrm{Nd}: \mathrm{YAG}}=9 \mathrm{~mm}$ single-crystal rod, nearly the same pump power is absorbed, leading therefore to an enhanced laser beam brightness as observed in Table 2.

Table 2. Numerically Calculated Laser Beam Quality Parameters with the Core-Doped Nd:YAG Ceramic Rod

\begin{tabular}{lcc}
\hline Resonator Configuration & Concave-Concave & Convex-Concave \\
\hline Mirror RoC (cm) & 500 & \\
Rear & 500 & -50 \\
Output & 80 & 500 \\
Resonator length (cm) & Laser beam quality & 80 \\
\multicolumn{1}{r}{} & 15.0 & 4.3 \\
$\mathrm{M}_{\mathrm{x}}^{2}$ & 15.1 & 4.4 \\
$\mathrm{M}_{\mathrm{y}}^{2}$ & 0.69 & 8.34 \\
Figure of merit B (W) & & \\
\hline
\end{tabular}

F7:1

F7:2

F7:3

F7:4 
Table 3. Nd:YAG Solar Laser Performances from Previous Nd:YAG Solar Lasers Compared to Modeled Output Using the Multi-Fresnel Lenses Pumping Approach

T3:1

$\mathrm{T} 3: 2$

T3:3

T3:4

T3:5

T3:6

T3:7

T3:8

T3:9

T3:10

T3:11

T3:12

\begin{tabular}{|c|c|c|c|c|c|c|}
\hline \multirow[b]{3}{*}{ Pumping configuration } & \multicolumn{2}{|c|}{ Previous Nd:YAG Solar Lasers } & \multicolumn{4}{|c|}{ Proposed Nd:YAG Solar Laser Approach } \\
\hline & Ref. [10] & Ref. [11] & \multicolumn{2}{|c|}{ Single-crystal } & \multicolumn{2}{|c|}{ Core-doped } \\
\hline & Side-pumping & End-pumping & \multicolumn{4}{|c|}{ Side-pumping } \\
\hline Resonator configuration & Concave-concave & Plane-concave & Concave-concave & Convex-concave & Concave-concave & Convex-concave \\
\hline Rod diameter $D_{\mathrm{Nd}: \mathrm{YAG}}(\mathrm{mm})$ & 4 & 6 & 9 & 9 & 8 & 8 \\
\hline Rod length (mm) & 30 & 100 & 30 & 30 & 30 & 30 \\
\hline \multicolumn{7}{|c|}{ Laser power } \\
\hline Laser power (W) & 24.7 & 120 & 146 & 146 & 157.8 & 158 \\
\hline Collection efficiency $\left(\mathrm{W} / \mathrm{m}^{2}\right)$ & 8.6 & 30 & 25.6 & 25.6 & 27.7 & 27.7 \\
\hline \multicolumn{7}{|c|}{ Laser beam quality } \\
\hline $\mathrm{M}_{\mathrm{x}}^{2} / \mathrm{M}_{\mathrm{y}}^{2}$ factors & $8.9 / 9.6$ & $137 / 137$ & $15.8 / 15.8$ & $6.0 / 6.1$ & $15.0 / 15.1$ & $4.3 / 4.4$ \\
\hline Figure of merit $\mathrm{B}(\mathrm{W})$ & 0.29 & 0.00639 & 0.59 & 4.0 & 0.69 & 8.34 \\
\hline
\end{tabular}

With the same resonator parameters as used for single-crystal rod, $0.69 \mathrm{~W}$ brightness figure of merit is expected with the concave-concave resonator configuration, which is $117 \%$ more than that of the $9 \mathrm{~mm}, 30 \mathrm{~mm}$ length single-crystal rod. By adopting the convex-concave resonator, the $M^{2}$ factors are reduced to $29 \%$ of their original values and the brightness figure of merit is therefore 12 times higher than that for concave-concave resonator. $8.34 \mathrm{~W}$ brightness figure of merit is numerically calculated, doubling the value of the single-crystal rod within the same resonator. It is also more than 1300 times higher than that of the most recent $120 \mathrm{~W} \mathrm{Nd}$ : YAG solar-pumped laser [11].

Table 3 summarizes the $\overline{\mathrm{Nd}}$ :YAG solar laser performances attained by both the multi-Fresnel lenses approach and the previous Nd:YAG solar lasers $[10,11]$.

\section{Preliminary Experimental Results}

High efficiency solar laser operation depends essentially on the successful concentration of the collected solar powers from all the narrow Fresnel lenses to a common focal spot, as shown in Figs. 8 and 9 , where

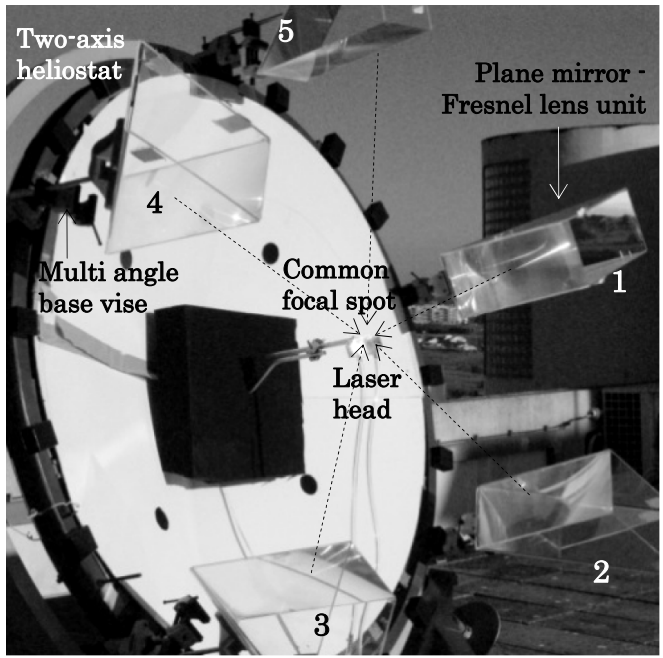

F8:1 Fig. 8. Five-fold plane mirror-Fresnel lens solar light collection F8:2 and concentration system. the Nd:YAG rod can be efficiently pumped within the fused silica cylindrical lens, as observed in Fig. 10. The implementation of the multiplane mirrorsFresnel lenses Nd:YAG solar laser system in Fig. 1 is both costly and time-consuming. We are expecting to test the whole system in less than two yearś time. The solar laser output performances can hopefully be reported in the future. Nevertheless, in order to validate our ZEMAX and LASCAD numerical analysis results in the previous sections, a five-fold plane mirror-Fresnel lens solar energy collection and concentration system has been built recently, as shown in Fig. 8. The main objective is to observe experimentally how well the concentrated solar power are focused into the Nd:YAG rod within the cylindrical lens. Since the total concentrated solar power is limited to only about $200 \mathrm{~W}$, no laser emission test has been carried out.

\section{A. Transmission Efficiency of Narrow Fresnel Lens}

Each plane-folding mirror $\left(M_{C}\right)$, with $0.57 \mathrm{~m}$ height and $0.16 \mathrm{~m}$ width, is front surface silver-coated with 95\% reflectivity. Each Fresnel lens $\left(F_{C}\right)$ has a narrow rectangular shape with $0.4 \mathrm{~m}$ height and $0.16 \mathrm{~m}$ width. The OHP Fresnel lenses are provided by Wuxi Bohai Optics Electronic Instrument Co., Ltd. Each plane folding mirror-Fresnel lens solar energy collection and concentration unit is built on an acrylic structure, as shown in Fig. 8. As predicted in the ZEMAX analysis, the narrow Fresnel lenses permit
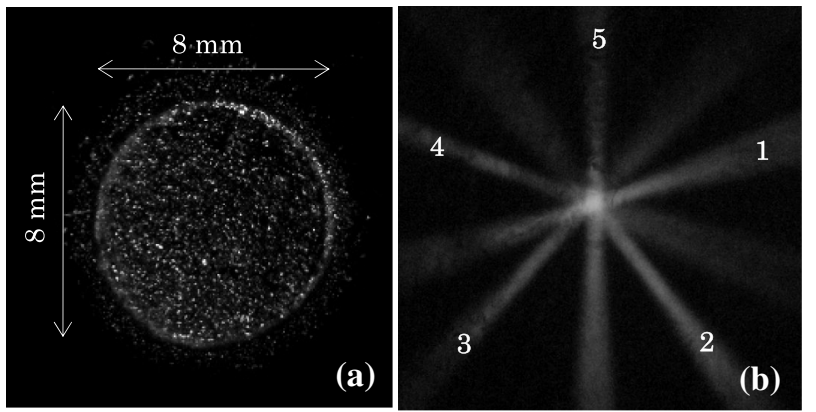

Fig. 9. (a) Focal spot of the single unit. (b) Concentrated solar radiations in the common focal zone. 


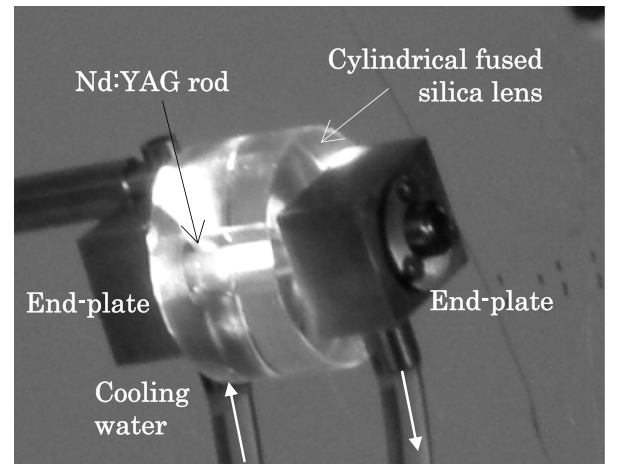

F10:1 Fig. 10. Concentrated solar radiation along the $6 \mathrm{~mm}$ diameter F10:2 Nd:YAG rod.

a tight paraxial focusing in the $x-y$ plane, while allowing some power spread along the $x-z$ plane. The significant difference in beam divergence in both $x-y$ plane and along the $x-z$ plane is clearly observed during the experiments. Incoming solar radiation is firstly concentrated into an $8 \mathrm{~mm}$ FWHM diameter focal spot by the narrow Fresnel lens via the plane folding mirror, as indicated by Fig. 9(a). Considering the terrestrial solar insolation of $930 \mathrm{~W} / \mathrm{m}^{2}$ for May 30,2013 , in the Lisbon area, $45.2 \mathrm{~W}$ solar power is measured at the focal spot of each unit, corresponding to the transmission efficiency of $80 \%$ for each Fresnel lens. This value is only slightly less than the ZEMAX numerical analysis result of $80.8 \%$. The total collected solar power from the five units can reach about $200 \mathrm{~W}$. The concentrated solar power is measured with a Molectron PowerMax 500D laser power meter with $2 \%$ measurement accuracy.

\section{B. Five-Fold Plane Mirror-Fresnel Lens Solar Energy Collection and Concentration System}

The five-fold plane mirror-Fresnel lens solar energy collection and concentration units, from 1 to 5 in Fig. 8 , are evenly mounted along the $0.83 \mathrm{~m}$ radius virtual cylinder around the center of a common focal spot. This formation has five-fold symmetry. Incoming solar radiation is firstly reflected by the plane folding mirrors $\left(M_{C}\right)$ to their respective Fresnel lenses $\left(F_{C}\right)$ for further concentration in the common focal zone, as shown in Fig. 9(b). The concentrated solar radiations are now successfully focused to the common focal spot, where the Nd:YAG laser head will be placed. The five plane mirror Fresnel lens solar energy collection and concentration units are individually mounted to a manual two-axis solar tracker, as shown in Fig. 8. Each solar energy collection and concentration unit is supported by an 83069 Stanley MaxSteel multiangle base vise, which allows a free angular alignment of each unit. The common focal spot in Fig. 9(b) has a FWHM diameter of about $10 \mathrm{~mm}$, slightly larger than the $8 \mathrm{~mm}$ FWHM focal diameter of each single unit, as shown in Fig. 9(a). Each multi-angle base vise also allow about $\pm \overline{30} \mathrm{~mm}$ free axial adjustment of the focal spot within the common focal zone. Therefore, the highest solar flux can be achieved at the common focal spot.
C. Optical Pumping Characteristics Along the Nd:YAG Rod

A high optical quality fused silica (99.999\%) cylindrical rod with $60 \mathrm{~mm}$ diameter is first cut to $30 \mathrm{~mm}$ length. To allow the efficient water cooling to the $1.1 \% \mathrm{Nd}$ :YAG laser rod, a $10 \mathrm{~mm}$ diameter cooling channel is drilled along the central axis of the fused silica cylindrical rod. The internal side surface of this channel is finally polished. The fused silica cylindrical lens with the cooling channel is then sandwiched between two end plates. The laser rod with $6 \mathrm{~mm}$ diameter by $50 \mathrm{~mm}$ length is finally mounted between the two endplates, as shown in Fig. 10. Cooling water circulates smoothly at 6 liter/min flow rate within the laser head. As indicated in Fig. 10, near uniform light distribution is eventually observed along the laser rod, revealing an excellent light focusing capability of the cylindrical lens with cooling water.

\section{Conclusions}

To largely improve the present day high-power solar laser beam quality, a three-stage multi-Fresnel lenses solar pumping scheme is proposed for the uniform side-pumping of either the Nd:YAG singlecrystal or the core-doped ceramic $\mathrm{Sm}^{3+} \mathrm{Nd}: \mathrm{YAG}$ rod, through either the cylindrical or the spherical lens. The best pumping conditions and optimum laser system parameters are found through ZEMAX and LASCAD numerical analysis, respectively. The $D_{\mathrm{Nd}: \mathrm{YAG}}=9 \mathrm{~mm}, L=30 \mathrm{~mm}$ single-crystal rod through the cylindrical lens offers the best compromise between laser power and pump absorption profile. $146 \mathrm{~W}$ multimode laser power is expected, corresponding to $25.6 \mathrm{~W} / \mathrm{m}^{2}$ collection efficiency. $0.59 \mathrm{~W}$ brightness figure of merit is numerically attained within the traditional concave-concave laser resonator, doubling the previous registered record. By adopting the convex-concave resonator, the $M^{2}$ factors can further be significantly reduced, resulting in $4.0 \mathrm{~W}$ brightness figure of merit. The use of coredoped Nd:YAG ceramic rods favors the production of more laser power. With the $D_{\mathrm{Nd} \text { YAG }}=8 \mathrm{~mm}, L=$ $20 \mathrm{~mm}$ core-doped rod through the spherical lens, $167 \mathrm{~W}$ laser power can be produced, leading to $29.3 \mathrm{~W} / \mathrm{m}^{2}$ collection efficiency, which approaches the record-high value for Nd:YAG solar laser. Very high brightness figure of merit of $8.34 \mathrm{~W}$ is numerically attained with the $D_{\mathrm{Nd} \text { :YAG }}=8 \mathrm{~mm}, L=30 \mathrm{~mm}$ core-doped rod within the convex-concave resonator, surpassing by more than 1300 times that of the mostrecent $120 \mathrm{~W}$ high-power Nd:YAG solar laser. The proposed solar-pumping approach can provide, in our opinion, an effective choice for attaining high solar laser power with good beam quality.

These research projects (PTDC/FIS/103599/2008 and PTDC/FIS/122420/2010) were funded by the Science and Technology Foundation of Portuguese Ministry of Science, Technology and Higher Education (FCT-MCTES). 


\section{References}

1. D. Graham-Rowe, "Solar-powered lasers," Nat. Photonics 4, 64-65 (2010).

2. C. W. Young, "A sun-pumped cw one-watt laser," Appl. Opt. 5, 993-997 (1966).

3. H. Arashi, Y. Oka, N. Sasahara, A. Kaimai, and M. Ishigame, "A solar-pumped cw 18 W Nd:YAG laser," Jpn. J. Appl. Phys. 23, 1051-1053 (1984).

4. M. Weksler and J. Shwartz, "Solar-pumped solid-state lasers," IEEE J. Quantum Electron. 24, 1222-1228 (1988).

5. V. Krupkin, J. A. Kagan, and A. Yogev, "Nonimaging optics and solar laser pumping at the Weizmann Institute," Proc. SPIE 2016, 50-60 (1993).

6. M. Lando, J. Kagan, B. Linyekin, and V. Dobrusin, "A solarpumped Nd:YAG laser in the high collection efficiency regime," Opt. Commun. 222, 371-381 (2003).

7. T. Yabe, S. Uchida, K. Ikuta, K. Yoshida, C. Baasandash, M. S. Mohamed, Y. Sakurai, Y. Ogata, M. Tuji, Y. Mori, Y. Satoh, T. Ohkubo, M. Murahara, A. Ikesue, M. Nakatsuka, T. Saiki, S. Motokoshi, and C. Yamanaka, "Demonstrated fossil-fuel-free energy cycle using magnesium and laser," Appl. Phys. Lett. 89, 261107 (2006).

8. T. Yabe, T. Ohkubo, S. Uchida, K. Yoshida, M. Nakatsuka, T. Funatsu, A. Mabuti, A. Oyama, K. Nakagawa, T. Oishi, K. Daito, B. Behgol, Y. Nakayama, M. Yoshida, S. Motokoshi, Y. Sato, and C. Baasandash, "High-efficiency and economical solar-energy-pumped laser with Fresnel lens and chromium co-doped laser medium," Appl. Phys. Lett. 90, 261120 (2007).

9. D. Liang and J. Almeida, "Highly efficient solar-pumped Nd: YAG laser," Opt. Express 19, 26399-26405 (2011).

10. J. Almeida, D. Liang, and E. Guillot, "Improvement in solarpumped Nd:YAG laser beam brightness," Opt. Laser Technol. 44, 2115-2119 (2012).

11. T. H. Dinh, T. Ohkubo, T. Yabe, and H. Kuboyama, "120 watt continuous wave solar-pumped laser with a liquid light-guide lens and a Nd:YAG rod," Opt. Lett. 37, 2670-2672 (2012).

12. D. Liang, J. Almeida, and E. Guillot, "Side-pumped continuouswave Cr:Nd:YAG ceramic solar laser," Appl. Phys. B (2013).

13. D. Liang and J. Almeida, "Design of ultrahigh brightness solar-pumped disk laser," Appl. Opt. 51, 6382-6388 (2012).
14. R. B. Chesler and D. Maydan, "Convex-concave resonators for $\mathrm{TEM}_{00}$ operation of solid-state ion lasers," J. Appl. Phys. 43, 2254-2257 (1972).

15. V. Magni, "Resonators for solid-state lasers with large-volume fundamental mode and high alignment stability," Appl. Opt. 25, 107-117 (1986).

16. P. H. Bernardes and D. Liang, "Solid-state laser pumping by light-guides," Appl. Opt. 45, 3811-3816 (2006).

17. ASTM Standard G173, (2012).

18. Z. Bin, C. Zhao, J. He, and S. Yang, "The study of active medium for solar-pumped solid-state lasers," Acta Opt. Sin. 27, 1-9 (2007).

19. Y. Abdel-Hadi, Development of Optical Concentrator Systems for Directly Solar Pumped Laser Systems (Verlag, 2005).

20. A. Ikesue, "Polycrystalline Nd:YAG ceramic lasers," Opt. Mater. 19, 183-187 (2002).

21. V. Lupei, "Ceramic laser materials and the prospect for high power lasers," Opt. Mater. 31, 701-706 (2009).

22. M. Ostermeyer and I. Brandenburg, "Simulation of the extraction of near diffraction limited Gaussian beams from side pumped core-doped ceramic Nd:YAG and conventional laser rods," Opt. Express 13, 10145-10156 (2005).

23. A. Sträßer and M. Ostermeyer, "Improving the brightness of side pumped power amplifiers by using core-doped ceramic rods," Opt. Express 14, 6687-6693 (2006).

24. J. Almeida and D. Liang, "Design of a high-brightness solarpumped laser by light-guides," Opt. Commun. 285, 5327-5333 (2012).

25. H. Yagi, J. F. Bisson, K. Ueda, and T. Yanagitani, "Y3Al5O12 ceramic absorbers for the suppression of parasitic oscillation in high-power Nd:YAG lasers," J. Lumin. 121, 88-94 (2006).

26. R. Huß, R. Wilhelm, C. Kolleck, J. Neumann, and D. Kracht, "Suppression of parasitic oscillations in a core-doped ceramic Nd:YAG laser by Sm:YAG cladding, “ Opt. Express 18, 13094-13101 (2010).

27. T. Denis, S. Hahn, S. Mebben, R. Wilhelm, C. Kolleck, J. Neumann, and D. Kracht, "Compact diode stack end pumped Nd:YAG amplifier using core doped ceramics," Appl. Opt. 49, 811-816 (2010). 


\section{Queries}

1. AU: Do you have a volume and page range for Ref. 12 ? 